\title{
A Novel High-order Linguistic Time Series Forecasting Model with the Growth of Declared Word-set
}

\author{
Nguyen Duy Hieu ${ }^{1}$ \\ Faculty of Natural Sciences and Technology \\ Tay Bac University \\ Son La, Vietnam
}

\author{
Pham Dinh Phong ${ }^{2 *}$ \\ Faculty of Information Technology \\ University of Transport and Communications \\ Hanoi, Vietnam
}

\begin{abstract}
The existing researches have shown that the fuzzy forecasting methods based on the high-order fuzzy time series are better than the fuzzy forecasting methods based on the firstorder fuzzy time series. The linguistic forecasting method based on the first-order linguistic time series which can handle directly the word-set of the linguistic variable have been examined by Hieu et al. This paper examines a novel model of high-order linguistic time series with the growth of declared word-set. A procedure for forecasting the enrollments of University of Alabama and Lahi crop production of India is developed based on the proposed model. In the proposed forecasting method, the high-order linguistic logical relationship groups will be established and utilized to calculate the forecasted values based on the quantitative semantics of the used words generated by the hedge algebras structure. The experimental results show that the forecasting accuracy of the proposed high-order forecasting method is better than their counterparts and the growth of the word-set of the linguistic variable is significant in increasing the accuracy of the forecasted results.
\end{abstract}

Keywords-Linguistic time series; high-order linguistic time series; linguistic logical relationship; hedge algebras; time series forecasting

\section{INTRODUCTION}

The subject of the time series forecasting model has attracted substantial attention in recent years. For almost the realistic cases, the forecasting problem is uncertainty. In other words, it seems impossible to predict the future values of things and phenomena with one hundred percent of accuracy. However, we can examine different forecasting models and find the best fit model for a certain dataset.

The living of human is a sequence of the decision-making process. In reality, they always use their linguistic experience to make decisions or in other words, they save their experiences of the real world by linguistic words and use them to make decisions in the future. In computer science, researchers have found many ways to simulate the human behaviors into the machines. The most successful one is fuzzy set theory approach. Fuzzy set theory proposed by Zadeh [1] uses fuzzy sets to represent the computational semantics of linguistic words. The utilization of fuzzy set theory to time series forecasting problem first proposed by Song and Chissom in 1993 [2]-[4]. For example, we can use linguistic words such as "small”, “quite small”, “very small”, "large”, "rather large”, "extremely large", etc. to describe the number of students who enroll to a university by years. By the fuzzy time series, the mentioned linguistic words can be represented by fuzzy sets. There are many publications of the fuzzy forecasting methods based on the fuzzy time series which can be found in the literature.

Chen in [5] examined the high-order fuzzy time series forecasting model where the $n^{\text {th }}$-order fuzzy logical relationship can be derived from the fuzzified historical data. In recent two decades, among so many improvements of the fuzzy time series forecasting methods, a number of high-order fuzzy time series forecasting methods has been proposed such as [6]-[23]. Among those studies, Singh [17] proposed a computational method of forecasting for the fuzzy time series model using high-order fuzzy logical relationships of differences of consecutive values. Most of other improvements of highorder fuzzy time are the proposals of the hybrid forecasting models with modern computational techniques using artificial neural networks [11], [12], [16], [19], machine learning [13], [14], particle swarm optimization [23], genetic algorithm [6], granular computing [8], etc.

Despite many important achievements, in the computation model of fuzzy time series, there is no formal connection between the fuzzy sets and the inherent semantics of the linguistic words represented by the fuzzy sets. In other words, the linguistic words are used in the fuzzy time series merely as the linguistic labels of fuzzy sets. Therefore, the relationships between the qualitative semantics of linguistic words and their fuzzy sets based semantics have not been thoroughly examined in the fuzzy time series forecasting models.

In the other side of natural language processing method, hedge algebras (HA) was introduced by Ho and Wechler in 1990 [24]. In hedge algebras, the linguistic variables can be modeled by an axiomatic way using inherent quantitative semantics of linguistic values. Many applications of hedge algebras can be found in the field of fuzzy control [25]-[31], data classification and regression using fuzzy rule-based systems [32], [33], image processing [34] and so on. In 2020, Hieu et al. [35], [36] proposed linguistic time series (LTS) forecasting model handling the linguistic words immediately based on the theory of hedge algebras. It is the first time that

*Corresponding Author

This research is funded by Ministry of Education and Training of Vietnam under a project led by Nguyen Duy Hieu from 2022 to 2023. 
the constructed linguistic time series can simulate the way human users describe numerical time series in terms of their linguistic words.

In this paper, inspired by the mentioned researches of fuzzy time series, we propose a novel high-order linguistic time series forecasting model. In the proposed model, the current linguistic value of the linguistic variable $x_{L}$ depend not only on the adjacent previous word but also the series of preceding words. In addition, the forecasting linguistic variable $\chi_{L}$ is considered as the linguistic counterpart of the numerical forecasting one $\chi_{N}$. So, the word-set of $\chi_{L}$ is infinite and just limited words of it are selected for a specific application, socalled declared word-set. In our proposed high-order LTS forecasting models, we assume that there is not any restriction on the declared word set. The accuracy of the proposed highorder LTS forecasting models can considerably increase when the declared word-set of $\chi_{L}$ grows. The proposed high-order LTS forecasting models are applied to the problems of the enrollments of University of Alabama [4] and the Lahi crop production of India [17] to illustrate the effectiveness of the proposed forecasting models and the scalability of the word-set of $\chi_{L}$. Besides, this study would like to present a comprehensive formalized methodology for handling linguistic words directly and simulate the way human users making a forecasting process in terms of their words. Therefore, we do not apply any optimization techniques which may improve the accuracy of forecasted results.

The rest of the paper will be organized as follows. Section II briefly introduces some fundamental knowledge of hedge algebras and the linguistic time series forecasting model. The proposed novel high-order linguistic time series forecasting model is also presented in this section. The experimental studies will be introduced and discussed in Section III. Finally, the paper will be covered with the conclusions.

\section{LingUistic TIME SERIES AND ITS FORECAsting MODEL}

\section{A. Some basic Knowledges of Hedge Algebras}

In the human society, people utilize their historical experiences to communicate one to another by linguistic words in natural language. Linguistic words themselves reveal their inherent semantics. Because of their qualitative semantics, every word has its semantic order relation with each other in the word-domain of a linguistic variable. That motivated Ho and Wechler [24], [37] to propose hedge algebras which can deal with linguistic words directly.

Let $\mathcal{A} \mathcal{X}=(\mathrm{X}, \mathrm{G}, \mathrm{C}, \mathrm{H} \leq)$ be a hedge algebras (HA), where $\mathrm{X}$ is the word-domain of a given linguistic variable $\mathcal{X}_{\mathrm{L}} ; \mathrm{G}$ is the set of two opposite generators, the negative one denoted by $\mathrm{c}^{-}$ and the positive one denoted by $\mathrm{c}^{+} ; \mathrm{C}$ is the set of three constants $0, \mathrm{~W}, 1$ which are the smallest word, the neutral and the largest ones (e.g., such specific words are "absolutely small", "medium" and "absolutely large"); $\mathrm{H}$ is the set of hedges such as "little”, "rather", "very", "extremely", and so on; $\leq$ is the semantic order relation in the word-domain.

Each linguistic value in HA structure has its own semantic. In order to model the quantitative semantics of all words in the word-domain, it is necessary to construct two notions of HA which are semantically quantifying mapping (SQM) and fuzziness measure, denoted by $v: X \rightarrow[0,1]$ and $\mathrm{m}: X \rightarrow$ $[0,1]$, respectively. SQM and fuzziness measure of $\mathrm{HA}$ structure can be defined as follows.

Definition 1. [38] A function $m: X \rightarrow[0,1]$ is said to be fuzziness measure of terms in $X$ provided that:

- $\mathrm{m}\left(c^{-}\right)+\mathfrak{m}\left(c^{+}\right)=1$;

- $\quad \sum_{h \in H} \mathfrak{m}(h u)=\mathfrak{m}(u)$, for $\forall u \in X$;

- $\mathfrak{m}(0)=\mathfrak{m}(W)=\mathfrak{m}(1)=0$, fuzziness measure of all constants is 0 ;

- For $\forall x, y \in X, \forall h \in H, \frac{\mathfrak{m}(h x)}{\mathfrak{m}(x)}=\frac{\mathfrak{m}(h y)}{\mathfrak{m}(y)}$, this proportion does not depend on any specific elements and, hence, it is called fuzziness measure of the hedge $h$ and denoted also by $\mu(h)$ for simplicity.

The fuzziness measure $m$ of a linguistic variable $\mathcal{X}_{L}$ has the following properties:

m1) $\mathfrak{m}(h x)=\mu(h) \mathfrak{m}(x)$, for $\forall x \in X$;

m2) $m\left(c^{-}\right)+m\left(c^{+}\right)=1$;

m3) $\sum_{-q \leq i \leq p, i \neq 0} \mathfrak{m}\left(h_{i} x\right)=\mathfrak{m}(x), \forall x \in X$;

m4) Set $\sum_{-q \leq i \leq-1} \mu\left(h_{i}\right)=\alpha, \sum_{1 \leq i \leq p} \mu\left(h_{i}\right)=\beta$, where $\alpha, \beta>0$ and $\alpha+\beta=1$.

In the computational model of HA, each linguistic word or hedge has its sign defined as follows.

Definition 2. [38] A function sign : $X \rightarrow\{-1,1\}$ is a mapping which is defined recursively as follows. For $h, h^{\prime} \in H$ and $c \in\left\{c^{-}, c^{+}\right\}$:

1) $\operatorname{sign}\left(c^{-}\right)=-1, \operatorname{sign}\left(c^{+}\right)=+1$;

2) $\operatorname{sign}(h c)=-\operatorname{sign}(c)(=+\operatorname{sign}(c))$ if $h$ is negative (positive) with respect to $c$;

3) $\operatorname{sign}\left(h^{\prime} h x\right)=-\operatorname{sign}(h x)$ if $h^{\prime} h x \neq h x$ and $h^{\prime}$ is negative with respect to $h$;

4) $\operatorname{sign}\left(h^{\prime} h x\right)=+\operatorname{sign}(h x)$ if $h^{\prime} h x \neq h x$ and $h^{\prime}$ is positive with respect to $h$.

Definition 3. [38] For given values of the fuzziness parameters of a variable $\mathcal{X}$, its corresponding SQM $v: X \rightarrow$ $[0,1]$ is defined as follows:

$$
\begin{aligned}
& v(W)=\theta=\mathrm{m}\left(c^{-}\right) ; \\
& v\left(c^{-}\right)=\theta-\alpha \mathfrak{m}\left(c^{-}\right) \text {and } v\left(c^{+}\right)=\theta+\alpha \mathfrak{m}\left(c^{+}\right) \\
& v\left(h_{j} x\right)=v(x)+\operatorname{sign}\left(h_{j} x\right)\left\{\sum_{i=\operatorname{sign}(j)}^{j} \mathfrak{m}\left(h_{i} x\right)-\right. \\
& \left.\omega\left(h_{j} x\right) \mathfrak{m}\left(h_{j} x\right)\right\}
\end{aligned}
$$

where

$\alpha)] \in\{\alpha, \beta\}$.

SQM and fuzziness measure are two important notions of HA. More specifically, for given $\alpha$ and $\theta$ parameters, all the components of the HA's computational model can be obtained. 


\section{B. Linguistic Time Series (LTS)}

Humans use their own language capacity to collect the data. Their brain saves all information in the real world by linguistic experience of specific owner. Human also use their own experience to make decision. In the time series forecasting problem, simulating the behavior of human's decision making process, the model also uses preceding data to predict the future value of time series data. When all the points of historical time series data are linguistic values, we have the LTS.

Definition 4. [35] Let $\mathbb{X}$ be a set of linguistic words in the natural language of a variable $\mathcal{X}$ defined on the universe of discourse $U_{x}$ to describe its numeric quantities. Then, any series $L(t), t=0,1,2, \ldots$, where $L(t)$ is a finite collection of words of $\mathbb{X}$, is called a linguistic time series.

Definition 5. [35] Let $\mathrm{T}$ be a LTS. In T, if every time $t=k-1$, the linguistic value of LTS is $x_{i}$ and $x_{j}$ is the linguistic value of $\mathrm{T}$ at the time $t=k$, then we have linguistic logical relationship of the form $x_{i} \rightarrow x_{j}$.

Definition 6. [35] Suppose that we have more than one linguistic logical relationship with the same left-hand side denoted by $x_{i} \rightarrow x_{j 1}, x_{i} \rightarrow x_{j 2}, \ldots, x_{i} \rightarrow x_{j n}$, then we can group them into the linguistic logical relationship group denoted by $x_{i} \rightarrow x_{j_{1}}, x_{j_{2}}, \ldots, x_{j_{n}}$.

In Def. 5, the logical relationship $x_{i} \rightarrow x_{j}$ indicates that the linguistic value $x_{j}$ to be caused by the only linguistic value $x_{i}$, called the first-order linguistic logical relationship. If there is more than one element on the left-hand side of the relationship, then we have the high-order linguistic logical relationship.

Definition 7. In the linguistic time series $\mathrm{T}$, if $x_{k}$, the linguistic value of $X$ at the time $k$, to be caused by $x_{k-1}, x_{k-2}$, $\ldots, x_{k-\lambda}$ are the linguistic values of $X$ at the time $k-1, k-$ $2, \ldots, k-\lambda$, respectively, then the linguistic logical relationship is presented by $x_{k-\lambda}, \ldots, x_{k-2}, x_{k-1} \rightarrow x_{k}$, socalled the $\lambda$ th-order linguistic logical relationship.

By Def. 7, we have first-order linguistic logical relationship when $\lambda=1$ and we have high-order linguistic logical relationship when $\lambda>1$.

In a similar way to Def. 6, the high-order linguistic logical relationship group is established based on the high-order linguistic logical relationships. Suppose that we have the highorder linguistic logical relationships:

$x_{k-\lambda}, \ldots, x_{k-2}, x_{k-1} \rightarrow x_{k 1}$,

$x_{k-\lambda}, \ldots, x_{k-2}, x_{k-1} \rightarrow x_{k 2}$,

$\ldots$,

$x_{k-\lambda}, \ldots, x_{k-2}, x_{k-1} \rightarrow x_{k m}$,

then we can group them into the high-order linguistic logical relationship group written in the form:

$x_{k-\lambda}, \ldots, x_{k-2}, x_{k-1} \rightarrow x_{k 1}, x_{k 2}, \ldots, x_{k m}$,

where $\lambda$ is the order of the linguistic logical relationship group.

\section{High-order LTS Forecasting Model}

Using the high-order linguistic logical relationship groups, the high-order LTS forecasting model was established and briefly reviewed as in Fig. 1.

In the first step, the universe of discourse and the HA structure with syntactic, qualitative and quantitative semantics are determined. The second step computes the numerical semantics of the declared words of $\mathcal{X}_{L}$. In the third step, according to the historical data, the high-order linguistic logical relationship groups, used in the next step, were observed. Using the linguistic logical relationship groups, ones can calculate the forecasted values of the time series data. Some performance measures can be utilized to assert the effective of the forecasting model.

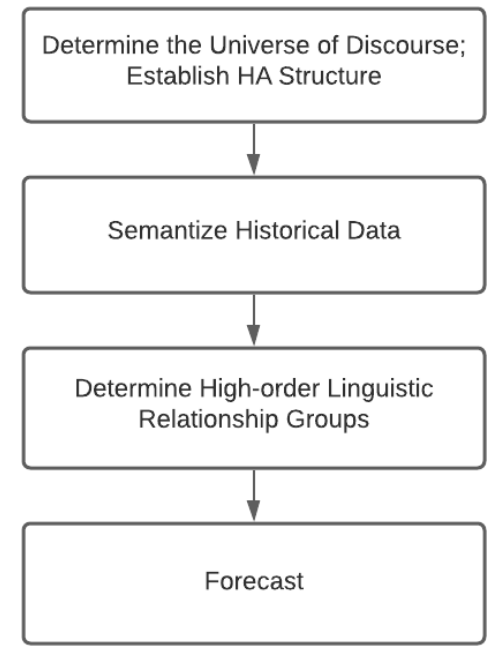

Fig. 1. The High-order Linguistic Time Series Forecasting Model.

\section{SOME EXPERIMENTAL RESULTS AND DISCUSSIONS}

This section presents the experimental results of the proposed high-order LTS forecasting models and shows their performance in comparison with their counterpart models. The comparative study is designed to compare the proposed highorder LTS forecasting model performance with those examined by Chen [5] and Singh [17], using the historical data of the enrollments of University of Alabama observed from 1971 to 1992, and Lahi crop production of India observed from 1981 to 2003.

The experiments are executed to show the following statements:

- The proposed high-order LTS forecasting models outperform their counterpart of fuzzy approaches with restricted linguistic words.

- In the proposed high-order LTS forecasting models, the forecasting linguistic variable $X_{L}$ is considered as the linguistic counterpart of the numerical forecasting one $x_{N}$. So, we assume that there is not any restriction on its declared word set's cardinality. The accuracy of the proposed high-order LTS forecasting models can considerably increase when the declared word-set of $x_{L}$ grows. 


\section{A. Forecast the Enrollments of University of Alabama}

1) Compare with the two counterparts examined by Chen and Singh: To show the efficiency of the proposed high-order LTS forecasting models, we apply them to the historical data of the enrollments of University of Alabama observed from 1971 to 1992, as shown the column "Actual Enrollment" in Table I. To be more convenient, the experiment follows the procedure as follows:

Step 1: Determine the syntactic, qualitative and quantitative semantics of the forecasting linguistic variable $\mathcal{X}$.

- The syntactic semantics are determined by declaring two primary words ${c^{-}}^{-}$small, $c^{+}=$large $(l)$ and the linguistic hedges. In this study, we just use two linguistic hedges $H^{-}=\{$Little $(L)\}$ and $H^{+}=\{$Very (V)\}. For the model illustration and comparative study, the cardinality of the declared word-set of $X_{L}$ is set to 9 (2-specificity level). So, we have: $U_{X, L}=X_{(2)}=\{\mathbf{0}$, $V \_s m a l l$, small, L_small, medium, L_large, large, V_large, $\mathbf{1}$ \}, where $\mathbf{0}$ stands for Absolutely small and $\mathbf{1}$ stands for Absolutely large.

- The qualitative semantics of $x_{L}$ is specified by the signs of hedges and the relative signs between them: $\operatorname{sign}(L)=\operatorname{sign}(L, L)=\operatorname{sign}(L, V)=-1$ and $\operatorname{sign}(V)=$ $\operatorname{sign}(V, V)=\operatorname{sign}(V, L)=+1$.

- The quantitative semantics is defined by specifying the fuzziness parameter values of $\mathcal{X}_{L}$. In this study, they are specified by human's experts and we have $m\left(c^{-}\right)=$ $0.437, \mu(L)=0.511$.

Step 2: Compute the numerical semantics of the declared words of $\mathcal{X}$.

Transform the numerical semantics (SQM-values) of the declared words in the normalized domain $[0,1]$ into the real numerical semantics in the universe of discourse $U_{\mathcal{X}}=[13000$, 20000] of $X_{L}$ by the following formula:

$v_{R}\left(l_{i}\right)=U_{X \text { min }}+\left(U_{X \max }-U_{X \text { min }}\right) \times v\left(l_{i}\right)$,

where $l_{i} \in X_{(2)}, v\left(l_{i}\right)$ is the numerical semantic of the word $l_{i}, U_{x_{\min }}$ is the lower bound and $U_{x_{\max }}$ is the upper bound of $U_{\mathcal{X}}$. Specifically, with the set $U_{\mathcal{X}, L}, m\left(c^{-}\right)=0.437$ and $\mu(L)=0.511$, we have the real numerical semantics of the declared words: $U_{\mathcal{X}, R}=\{13000,13731,14496,15295,16059$, 17044, 18073, 19058, 20000\}.

Step 3: Generate LTS from the given numerical time series.

Based on the real numerical semantics, the historical data of the enrollments are transformed into the linguistic words selected from the declared word-set of $X_{\mathrm{L}}$ in such a way that each historical data value is assigned a linguistic word which has its real numerical semantics closest to that historical data value at that timestamp. For example, the enrollment value of 15460 is of the year of 1975 closest to the real numerical semantics of 15295 of the word Little small in $\mathrm{U}_{\mathcal{X}, \mathrm{L}}$. Therefore, that enrollment value is assigned the word Little small. By doing the same way for the enrollment data of other years, we obtain the LTS as shown in the column "Constructed LTS" in
Table I. Once the LTS is constructed, the high-order linguistic logical relationships (LLRs) and the high-order linguistic logical relationship groups (LLRGs) are created. Just for the illustration of the case of 2-specificity level of $x_{\mathrm{L}}$ (9 declared words), the second-order and third-order LLRGs are shown in Table II and Table III, respectively.

TABLE I. THE LINGUISTIC TIME SERIES OF THE ENROLLMENTS OF THE UNIVERSITY OF ALABAMA FROM 1971 TO 1992 USING 9 WORDS

\begin{tabular}{|c|c|c|}
\hline Year & Actual Enrollment & Constructed LTS \\
\hline 1971 & 13055 & Absolutely small \\
\hline 1972 & 13563 & Very small \\
\hline 1973 & 13867 & Very small \\
\hline 1974 & 14696 & small \\
\hline 1975 & 15460 & Little small \\
\hline 1976 & 15311 & Little small \\
\hline 1977 & 15603 & Little small \\
\hline 1978 & 15861 & medium \\
\hline 1979 & 16807 & Little large \\
\hline 1980 & 16919 & Little large \\
\hline 1981 & 16388 & medium \\
\hline 1982 & 15433 & Little small \\
\hline 1983 & 15497 & Little small \\
\hline 1984 & 15145 & Little small \\
\hline 1985 & 15163 & Little small \\
\hline 1986 & 15984 & medium \\
\hline 1987 & 16859 & Little large \\
\hline 1988 & 18150 & large \\
\hline 1989 & 18970 & Very large \\
\hline 1990 & 19328 & Very large \\
\hline 1991 & 19337 & Very large \\
\hline 1992 & 18876 & Very large \\
\hline
\end{tabular}

TABLE II. THE SECOND-ORDER LLRGS USING 9 WORDS

\begin{tabular}{|c|c|}
\hline Group & 2-order LRRG \\
\hline 1 & $V \_s m a l l, V \_s m a l l ~ \rightarrow V \_s m a l l$ \\
\hline 2 & V_small, V_small $\rightarrow$ small \\
\hline 3 & small, $V \_s m a l l ~ \rightarrow L \_s m a l l$ \\
\hline 4 & L_small, small $\rightarrow L \_s m a l l$ \\
\hline 5 & L_small, $L \_s m a l l ~ \rightarrow L$ L_small, medium \\
\hline 6 & medium, L_small $\rightarrow$ L_large \\
\hline 7 & L_large, medium $\rightarrow$ L_large, large \\
\hline 8 & L_large, $L \_$large $\rightarrow$ medium \\
\hline 9 & medium, L_large $\rightarrow$ L_small \\
\hline 10 & L_small, medium $\rightarrow L \_s m a l l$ \\
\hline 11 & large, L_large $\rightarrow V$ _large \\
\hline 12 & V_large, large $\rightarrow$ V_large \\
\hline 13 & V_large, V_large $\rightarrow$ V_large \\
\hline
\end{tabular}


TABLE III. THE THIRD-ORDER LLRGS USING 9 WORDS

\begin{tabular}{|c|c|}
\hline Group & 3-order LRRG \\
\hline 1 & V_small, $V \_s m a l l, A \_s m a l l ~ \rightarrow$ small \\
\hline 2 & small, $V \_s m a l l, V \_s m a l l ~ \rightarrow L \_s m a l l$ \\
\hline 3 & L_small, small, V_small $\rightarrow$ L_small \\
\hline 4 & L_small, L_small, small $\rightarrow$ L_small \\
\hline 5 & L_small, L_small, $L \_s m a l l ~ \rightarrow$ medium, $L \_s m a l l$ \\
\hline 6 & medium, $L \_s m a l l, L \_s m a l l ~ \rightarrow L$ L_large \\
\hline 7 & L_large, medium, L_small $\rightarrow$ L_large, large \\
\hline 8 & L_large, $L \_l a r g e$, medium $\rightarrow$ medium \\
\hline 9 & medium, $L \_$large, $L \_$large $\rightarrow L \_$_small \\
\hline 10 & L_small, medium, $L \_l a r g e ~ \rightarrow L \_s m a l l$ \\
\hline 11 & L_small, L_small, medium $\rightarrow L$ L_small \\
\hline 12 & large, $L \_$large, medium $\rightarrow V \_$_large \\
\hline 13 & V_large, large, $L \_$large $\rightarrow V$ _large \\
\hline 14 & V_large, $V$ _large, large $\rightarrow$ V_large \\
\hline 15 & V_large, $V$ _large, $V$ _large $\rightarrow V$ _large \\
\hline
\end{tabular}

Step 4: Calculate the crisp forecasted values. Two applied computation rules are as follows:

1) If an high-order LLRG is of the form: $l_{i 1}, l_{i 2}, \ldots, l_{\lambda} \rightarrow$ $l_{j 1}, l_{j 2}, \ldots, l_{j p}(\lambda, p \geq 1)$, where $l_{i 1}, l_{i 2}, \ldots, l_{\lambda}$ is the current state of a year, say $\mathrm{k}$ and $v_{R}\left(l_{j 1}\right), v_{R}\left(l_{j 2}\right), \ldots, v_{R}\left(l_{j p}\right)$ are the real numerical semantics of the words $l_{j 1}, l_{j 2}, \ldots, l_{j p}$, respectively, then the numeric forecasted value of year $k+1$ is $\left(v_{R}\left(l_{j 1}\right)+\right.$ $\left.v_{R}\left(l_{j 2}\right)+\cdots+v_{R}\left(l_{j p}\right)\right) / p$.

2) If the current state of year $k$ is $l_{i 1}, l_{i 2}, \ldots, l_{\lambda}$ and there does not exist any word in the right-hand side of any high- order LLRG, then, the forecasted value of year $k+1$ is $\left(v_{R}\left(l_{i 1}\right)+v_{R}\left(l_{i 2}\right)+\cdots+v_{R}\left(l_{i p}\right)\right) / \lambda$.

In this study, the mean square error (MSE) and average forecasting error $(A F E)$ are used to evaluate the forecasting models, where $N$ is the number of forecasted value, $F_{i}$ and $A_{i}$ are the forecasted value and the actual value, respectively; $\epsilon_{i}$ is forecasting error of year $i$ :

$$
\begin{aligned}
& M S E=\left(\frac{1}{N}\right) \sum_{i=1}^{N}\left(F_{i}-A_{i}\right)^{2} \\
& \epsilon_{i}(\text { in percent })=\frac{\left|F_{i}-A_{i}\right|}{A_{i}} \times 100 \\
& A F E(\text { in percent })=\frac{\epsilon_{i}}{N}
\end{aligned}
$$

The forecasted enrollments by applying the proposed highorder (from 2-order to 9-order) LTS forecasting models using 9 declared words ( 9 words selected from the word-set of $\mathcal{X}_{\mathrm{L}}$ for describing the universe of discourse $\mathrm{U}_{x}$ ) are shown in Table IV. The comparison of MSE values with three other high-order fuzzy time series forecasting models are shown in Table V. The MSE values of the proposed high-order LTS forecasting models are much better than all their counterparts (Chen [5], Hwang et al. [39] and Singh [17]) from the 4-order to 9-order. Evaluated based on AFE values, it is easy to see in Table VI that all proposed high-order LTS forecasting models have AFE values smaller than their counterparts (Song and

\begin{tabular}{|c|c|c|c|c|c|c|c|c|c|c|c|c|c|c|c|c|c|}
\hline \multirow[b]{2}{*}{ Year } & \multirow{2}{*}{$\begin{array}{l}\text { Actu. } \\
\text { Enrol. }\end{array}$} & \multicolumn{8}{|c|}{ Proposed (9 declared words) } & \multicolumn{8}{|c|}{ Proposed (17 declared words) } \\
\hline & & \begin{tabular}{|l|}
$2-$ \\
order \\
\end{tabular} & \begin{tabular}{|l|}
$3-$ \\
order
\end{tabular} & \begin{tabular}{|l|}
$4-$ \\
order
\end{tabular} & \begin{tabular}{|l|}
$5-$ \\
order
\end{tabular} & \begin{tabular}{|l|}
$6-$ \\
order
\end{tabular} & \begin{tabular}{|l|}
$7-$ \\
order
\end{tabular} & \begin{tabular}{|l|}
$\begin{array}{l}8- \\
\text { order }\end{array}$ \\
\end{tabular} & $\begin{array}{l}9- \\
\text { order }\end{array}$ & \begin{tabular}{|l|}
$2-$ \\
order
\end{tabular} & \begin{tabular}{|l|}
$3-$ \\
order
\end{tabular} & \begin{tabular}{|l|}
$4-$ \\
order
\end{tabular} & \begin{tabular}{|l|}
$5-$ \\
order
\end{tabular} & \begin{tabular}{|l|}
$6-$ \\
order
\end{tabular} & \begin{tabular}{|l|}
$7-$ \\
order
\end{tabular} & \begin{tabular}{|l|}
$8-$ \\
order
\end{tabular} & \begin{tabular}{|l|}
$9-$ \\
order
\end{tabular} \\
\hline 1971 & 13055 & & & & & & & & & & & & & & & & \\
\hline 1972 & 13563 & & & & & & & & & & & & & & & & \\
\hline 1973 & 13867 & 13731 & & & & & & & & 13750 & & & & & & & \\
\hline 1974 & 14696 & 14496 & 14496 & & & & & & & 14644 & 14644 & & & & & & \\
\hline 1975 & 15460 & 15295 & 15295 & 15295 & & & & & & 15537 & 15537 & 15537 & & & & & \\
\hline 1976 & 15311 & 15295 & 15295 & 15295 & 15295 & & & & & 15169 & 15169 & 15169 & 15169 & & & & \\
\hline 1977 & 15603 & 15677 & 15295 & 15295 & 15295 & 15295 & & & & 15353 & 15537 & 15537 & 15537 & 15537 & & & \\
\hline 1978 & 15861 & 15677 & 15677 & 16059 & 16059 & 16059 & 16059 & & & 15795 & 15795 & 15795 & 15795 & 15795 & 15795 & & \\
\hline 1979 & 16807 & 17044 & 17044 & 17044 & 17044 & 17044 & 17044 & 17044 & & 16689 & 16689 & 16689 & 16689 & 16689 & 16689 & 16689 & \\
\hline 1980 & 16919 & 17558 & 17558 & 17558 & 17558 & 17044 & 17044 & 17044 & 17044 & 16689 & 16689 & 16689 & 16689 & 16689 & 16689 & 16689 & 16689 \\
\hline 1981 & 16388 & 16059 & 16059 & 16059 & 16059 & 16059 & 16059 & 16059 & 16059 & 16164 & 16164 & 16164 & 16164 & 16164 & 16164 & 16164 & 16164 \\
\hline 1982 & 15433 & 15295 & 15295 & 15295 & 15295 & 15295 & 15295 & 15295 & 15295 & 15537 & 15537 & 15537 & 15537 & 15537 & 15537 & 15537 & 15537 \\
\hline 1983 & 15497 & 15295 & 15295 & 15295 & 15295 & 15295 & 15295 & 15295 & 15295 & 15537 & 15537 & 15537 & 15537 & 15537 & 15537 & 15537 & 15537 \\
\hline 1984 & 15145 & 15677 & 15295 & 15295 & 15295 & 15295 & 15295 & 15295 & 15295 & 15169 & 15169 & 15169 & 15169 & 15169 & 15169 & 15169 & 15169 \\
\hline 1985 & 15163 & 15677 & 15677 & 15295 & 15295 & 15295 & 15295 & 15295 & 15295 & 15353 & 15169 & 15169 & 15169 & 15169 & 15169 & 15169 & 15169 \\
\hline 1986 & 15984 & 15677 & 15677 & 16059 & 16059 & 16059 & 16059 & 16059 & 16059 & 16164 & 16164 & 16164 & 16164 & 16164 & 16164 & 16164 & 16164 \\
\hline 1987 & 16859 & 17044 & 17044 & 17044 & 17044 & 17044 & 17044 & 17044 & 17044 & 16689 & 16689 & 16689 & 16689 & 16689 & 16689 & 16689 & 16689 \\
\hline 1988 & 18150 & 17558 & 17558 & 17558 & 17558 & 18073 & 18073 & 18073 & 18073 & 18053 & 18053 & 18053 & 18053 & 18053 & 18053 & 18053 & 18053 \\
\hline 1989 & 18970 & 19058 & 19058 & 19058 & 19058 & 19058 & 19058 & 19058 & 19058 & 18855 & 18855 & 18855 & 18855 & 18855 & 18855 & 18855 & 18855 \\
\hline 1990 & 19328 & 19058 & 19058 & 19058 & 19058 & 19058 & 19058 & 19058 & 19058 & 19327 & 19327 & 19327 & 19327 & 19327 & 19327 & \begin{tabular}{|l|}
19327 \\
\end{tabular} & 19327 \\
\hline 1991 & 19337 & 19058 & 19058 & 19058 & 19058 & 19058 & 19058 & 19058 & 19058 & 19327 & 19327 & 19327 & 19327 & 19327 & 19327 & 19327 & 19327 \\
\hline 1992 & 18876 & 19058 & 19058 & 19058 & 19058 & 19058 & 19058 & 19058 & 19058 & 18855 & 18855 & 18855 & 18855 & 18855 & 18855 & 18855 & 18855 \\
\hline \multicolumn{2}{|l|}{ MSE } & \begin{tabular}{|l|l|}
98783 \\
\end{tabular} & 94001 & 78665 & 81691 & \begin{tabular}{|l|l|}
40704 \\
\end{tabular} & 37093 & 36943 & 35464 & \begin{tabular}{|l|l|}
17996 \\
\end{tabular} & 13264 & 13851 & 14313 & 13951 & 14591 & 15322 & 15429 \\
\hline \multicolumn{2}{|l|}{ AFE } & 1.60 & 1.58 & 1.37 & 1.39 & 1.11 & 1.05 & 1.03 & 1.0 & 0.69 & 0.56 & 0.57 & 0.57 & 0.55 & 0.56 & 0.57 & 0.56 \\
\hline
\end{tabular}
Chissom [4], Hwang et al. [39] and Singh [17]).

TABLE IV. FORECASTED ENROLLMENTS BY PROPOSED HIGH-ORDER LTS FORECASTING MODELS USING 9 AND 17 DECLARED WORDS. 
TABLE V. COMPARISON OF MSE VALUES

\begin{tabular}{|l|l|l|l|l|l|l|l|l|}
\hline Methods/MSE & 2-order & $\mathbf{3 - o r d e r}$ & 4-order & 5-order & 6-order & 7-order & $\mathbf{8}$-order & 9-order \\
\hline Chen [5] & $\mathbf{8 9 , 0 9 3}$ & $\mathbf{8 6 , 6 9 4}$ & 89,376 & 94,539 & 98,215 & 104,056 & 102,179 & 102,789 \\
\hline Hwang et al. [39] & 333,171 & 299,634 & 315,489 & 278,919 & 296,950 & 316,720 & 301,228 & 306,485 \\
\hline Singh [17] & 119,189 & 97,180 & 126,676 & 113,421 & 163,137 & 148,618 & 169,149 & 123,964 \\
\hline Proposed (9 declared words) & 98,783 & 94,001 & $\mathbf{7 8 , 6 6 5}$ & $\mathbf{8 1 , 6 9 1}$ & $\mathbf{4 0 , 7 0 4}$ & $\mathbf{3 7 , 0 9 3}$ & $\mathbf{3 6 , 9 4 3}$ & $\mathbf{3 5 , 4 6 4}$ \\
\hline
\end{tabular}

TABLE VI. COMPARISON OF AVERAgE ERROR (AFE) OF FORECASTS

\begin{tabular}{|l|l|l|l|l|l|l|l|l|}
\hline Methods/MSE & 2-order & 3-order & 4-order & 5-order & 6-order & 7-order & 8-order & 9-order \\
\hline Song and Chissom [4] & 3.15 & 3.89 & 4.37 & 4.41 & 4.49 & 4.35 & 4.45 & 4.23 \\
\hline Hwang et al. [39] & 2.99 & 2.94 & 3.12 & 2.92 & 3.01 & 3.08 & 2.89 & 2.79 \\
\hline Singh [17] & 1.80 & 1.56 & 1.74 & 1.68 & 2.07 & 1.89 & 1.98 & 1.65 \\
\hline Proposed (9 declared words) & $\mathbf{1 . 6 0}$ & $\mathbf{1 . 5 8}$ & $\mathbf{1 . 3 7}$ & $\mathbf{1 . 3 9}$ & $\mathbf{1 . 1 1}$ & $\mathbf{1 . 0 5}$ & $\mathbf{1 . 0 3}$ & $\mathbf{1 . 0}$ \\
\hline
\end{tabular}

3) The accuracy of the proposed high-order LTS forecasting models increases by allowing the current used word-set of the forecasting linguistic variable to grow.

A special feature of the LTS forecasting models is that it considers the forecasting linguistic variable $\chi_{L}$ as the linguistic counterpart of the numerical forecasting one. So, there is not any restriction on its declared word set's cardinality. This experimental study will show that the forecasting accuracy will considerably increase when the declared word-set of $x_{L}$ grows. In HA, the word-set of $\chi_{L}$ are generated automatically by acting the linguistic hedges on two generator words [24], [37]. The maximum length of words is limited by an integer $k$, so-called $k$-specificity level. For example, in Step 1 of the proposed high-order forecasting model described above, we use two linguistic hedges $L$ and $V$ acting on two generator words, so when $k=1,2,3,4$ and 5, we have 5, 9, 17, 33, 65 generated words, respectively. To show the scalability of the word-set of $\mathcal{X}_{L}$, we assume that its cardinality grows from 9 to 17,33 and 65 words respectively corresponding to 3-, 4- and 5specificity levels. For each declared word-set cardinality, the fuzziness parameter values of $\chi_{L}$ should be adjusted suitably by human's experts as shown in Table VII. For example, in case of 17 declared words, the value of $m\left(c^{-}\right)$is 0.527 and the values of $\mu(L)$ is 0.412 , etc.

Intuitively seen in Table IV and Table VIII, with the same order, the greater used linguistic words, the smaller corresponding MSE and AFE values we will have. Specifically, the 9-order LTS forecasting model using 65 words has its MSE value of 283 and its AFE value of 0.07 , better than the respective ones of the other models with same order using 33, 17 and 9 words in turn, respectively, 1023 and 1.4, 15429 and 0.6, 35464 and 1.0. We also have the same analysis results for other order (from 2 to 8) using the same words. These comparison results state that the forecasting accuracy will considerably increase when the declared wordset of $\mathcal{X}_{L}$ grows (from 9 words to 65 words) and show the effectiveness of the proposed high-order linguistic forecasting method.

\section{B. Forecast the Lahi Crop Production of Indian}

To prove that the proposed high-order LTS forecasting models are much more efficient than the ones proposed by Singh in [17], the proposed models are applied to forecast the Lahi crop production (productivity in kg per hectare) from the farm of G.B. Pant University, Pantnagar, India, observed from 1981 to 2003 [17]. With the observed production values are from 440 and 1067, we can set the value interval of the universe of discourse of the variable $X_{L}$ to be $U_{\mathcal{X}}=[400$, 1100] as in [17]. Two primary words are $c^{-}=\operatorname{low}(l)$ and $c^{+}=\operatorname{high}(h)$ and two linguistic hedges are Little $(L) \in \mathrm{H}^{-}$ and $\operatorname{Very}(V) \in H^{+}$. For illustration, the declared word-set of $x_{L}$ in case of 2-specificity level is $U_{X, L}=X_{(2)}=\{0$, Very low, low, Little low, medium, Little high, high, Very high, 1$\}$. The fuzziness parameter values are chosen by human's experts as $m\left(c^{-}\right)=m($ low $)=0.5$ and $\mu(L)=0.51$ for all various orders and declared words. So, the real numerical semantics of the declared words are $U_{\chi, R}=\{400,484,571.5,662.5,750,837.5$, 928.5, 1016, 1100\}. Based on the given data setting, transform the observed historical data of the Lahi crop production shown the column "Actual production" in Table IX to the linguistic time series, establish the high-order LLRs and the high-order LLRGs, finally calculate the crisp forecasted values. The crop production forecasted results of the various high-order (from 2 to 9) LTS forecasting models are shown Table IX.

The comparisons of MSE and AFE values between the proposed LTS forecasting models with various orders using nine words and the corresponding ones proposed by Singh in [8] are shown in Table $X$. Indeed, the comparison results prove that the proposed high-order LTS forecasting models are much better than the ones proposed by Singh on both evaluation methods of MSE and AFE.

TABLE VII. FUZZINESS PARAMETER VALUES

\begin{tabular}{|l|l|l|l|l|l|}
\hline \multicolumn{2}{|l|}{17 words } & \multicolumn{2}{l|}{ 33 words } & 65 words \\
\hline $\boldsymbol{m}\left(\boldsymbol{c}^{-}\right)$ & $\boldsymbol{\mu ( L )}$ & $\boldsymbol{m ( c ^ { - } )}$ & $\boldsymbol{\mu ( L )}$ & $\boldsymbol{m}\left(\boldsymbol{c}^{-}\right)$ & $\boldsymbol{\mu ( L )}$ \\
\hline 0.527 & 0.412 & 0.65 & 0.35 & 0.65 & 0.35 \\
\hline
\end{tabular}


TABLE VIII. Forecasted ENROLLMENTS By PROPOSED High-ORDER LTS ForeCASTING MODELS USING 33 AND 65 DECLARED WORDS

\begin{tabular}{|c|c|c|c|c|c|c|c|c|c|c|c|c|c|c|c|c|c|}
\hline \multirow[b]{2}{*}{ Year } & \multirow{2}{*}{$\begin{array}{l}\text { Actu. } \\
\text { Enrol. }\end{array}$} & \multicolumn{8}{|c|}{ Proposed (33 declared words) } & \multicolumn{8}{|c|}{ Proposed (65 declared words) } \\
\hline & & $\begin{array}{l}2- \\
\text { order }\end{array}$ & $\begin{array}{l}\text { 3- } \\
\text { order }\end{array}$ & $\begin{array}{l}4- \\
\text { order }\end{array}$ & $\begin{array}{l}\text { 5- } \\
\text { order }\end{array}$ & \begin{tabular}{|l|}
$6-$ \\
order \\
\end{tabular} & $\begin{array}{l}\text { - } \\
\text { order }\end{array}$ & \begin{tabular}{|l|}
$8-$ \\
order
\end{tabular} & \begin{tabular}{|l|}
$9-$ \\
order
\end{tabular} & $\begin{array}{l}2- \\
\text { order }\end{array}$ & $\begin{array}{l}\text { 3- } \\
\text { order }\end{array}$ & \begin{tabular}{|l|} 
order \\
orde
\end{tabular} & $\begin{array}{l}\text { 5- } \\
\text { order }\end{array}$ & \begin{tabular}{|l|}
$6-$ \\
order
\end{tabular} & \begin{tabular}{|l|}
$7-$ \\
order
\end{tabular} & \begin{tabular}{|l|}
$8-$ \\
order
\end{tabular} & \begin{tabular}{|l|}
$9-$ \\
order
\end{tabular} \\
\hline 1971 & 13055 & & & & & & & & & & & & & & & & \\
\hline 1972 & 13563 & & & & & & & & & & & & & & & & \\
\hline 1973 & 13867 & 13812 & & & & & & & & 13812 & & & & & & & \\
\hline 1974 & 14696 & 14485 & 14485 & & & & & & & 14638 & 14638 & & & & & & \\
\hline 1975 & 15460 & 15520 & 15520 & 15520 & & & & & & 15438 & 15438 & 15438 & & & & & \\
\hline 1976 & 15311 & 15285 & 15285 & 15285 & 15285 & & & & & 15285 & 15285 & 15285 & 15285 & & & & \\
\hline 1977 & 15603 & 15520 & 15520 & 15520 & 15520 & 15520 & & & & 15673 & 15673 & 15673 & 15673 & 15673 & & & \\
\hline 1978 & 15861 & 15958 & 15958 & 15958 & 15958 & 15958 & 15958 & & & 15958 & 15958 & 15958 & 15958 & 15958 & 15958 & & \\
\hline 1979 & 16807 & 16750 & 16750 & 16750 & 16750 & 16750 & 16750 & 16750 & & 16795 & 16795 & 16795 & 16795 & 16795 & 16795 & 16795 & \\
\hline 1980 & 16919 & 16877 & \begin{tabular}{|l|}
16877 \\
\end{tabular} & 16877 & 16877 & 16877 & 16877 & 16877 & 16877 & 16877 & 16877 & 16877 & 16877 & \begin{tabular}{|l|}
16877 \\
\end{tabular} & 16877 & \begin{tabular}{|l|}
16877 \\
\end{tabular} & 16877 \\
\hline 1981 & 16388 & 16388 & 16388 & 16388 & 16388 & 16388 & 16388 & 16388 & 16388 & 16388 & 16388 & 16388 & 16388 & 16388 & 16388 & 16388 & 16388 \\
\hline 1982 & 15433 & 15520 & 15520 & 15520 & 15520 & 15520 & 15520 & 15520 & 15520 & 15438 & 15438 & 15438 & 15438 & 15438 & 15438 & 15438 & 15438 \\
\hline 1983 & 15497 & 15520 & 15520 & 15520 & 15520 & 15520 & 15520 & 15520 & 15520 & 15520 & 15520 & 15520 & 15520 & 15520 & 15520 & 15520 & 15520 \\
\hline 1984 & 15145 & 15158 & 15158 & 15158 & 15158 & 15158 & 15158 & 15158 & 15158 & 15158 & 15158 & 15158 & 15158 & 15158 & 15158 & 15158 & 15158 \\
\hline 1985 & 15163 & 15158 & 15158 & 15158 & 15158 & 15158 & 15158 & 15158 & 15158 & 15158 & 15158 & 15158 & 15158 & 15158 & 15158 & 15158 & 15158 \\
\hline 1986 & 15984 & 15958 & 15958 & 15958 & 15958 & 15958 & 15958 & 15958 & 15958 & 15958 & 15958 & 15958 & 15958 & 15958 & 15958 & 15958 & 15958 \\
\hline 1987 & 16859 & 16877 & 16877 & 16877 & 16877 & 16877 & 16877 & 16877 & 16877 & 16877 & 16877 & 16877 & 16877 & 16877 & 16877 & 16877 & 16877 \\
\hline 1988 & 18150 & 18176 & 18176 & 18176 & 18176 & 18176 & 18176 & 18176 & 18176 & 18152 & 18152 & 18152 & 18152 & 18152 & 18152 & 18152 & 18152 \\
\hline 1989 & 18970 & 18965 & 18965 & \begin{tabular}{|l|}
18965 \\
\end{tabular} & 18965 & 18965 & 18965 & 18965 & 18965 & 18965 & 18965 & 18965 & 18965 & 18965 & 18965 & 18965 & 18965 \\
\hline 1990 & 19328 & 19327 & 19327 & 19327 & 19327 & 19327 & 19327 & 19327 & 19327 & 19327 & 19327 & 19327 & 19327 & 19327 & 19327 & 19327 & \begin{tabular}{|l|l|}
19327 \\
\end{tabular} \\
\hline 1991 & 19337 & 19327 & 19327 & 19327 & 19327 & 19327 & 19327 & 19327 & 19327 & 19327 & 19327 & 19327 & 19327 & \begin{tabular}{|l|}
19327 \\
\end{tabular} & 19327 & 19327 & 19327 \\
\hline 1992 & 18876 & 18838 & 18838 & 18838 & 18838 & 18838 & 18838 & 18838 & 18838 & 18882 & 18882 & 18882 & 18882 & 18882 & 18882 & 18882 & 18882 \\
\hline \multicolumn{2}{|l|}{ MSE } & 4234 & 4297 & 2062 & 1972 & 2053 & 1731 & 1182 & 1023 & 1284 & 1192 & 1078 & 1106 & 1133 & 882 & 273 & 283 \\
\hline \multicolumn{2}{|l|}{ AFE } & 0.28 & 0.28 & 0.21 & 0.20 & 0.20 & 0.18 & 0.15 & 0.14 & 0.16 & 0.15 & 0.13 & 0.13 & 0.13 & 0.11 & 0.07 & 0.07 \\
\hline
\end{tabular}

TABLE IX. ForeCAsted Productions By Proposed High-ORder LTS ForECASTING MODELS USING 9 DECLARED WORDS

\begin{tabular}{|c|c|c|c|c|c|c|c|c|c|c|}
\hline Year & \begin{tabular}{|l|} 
Actual \\
production
\end{tabular} & \begin{tabular}{|l|} 
Linguistic \\
time series
\end{tabular} & 2-order & 3-order & 4-order & 5-order & 6-order & 7-order & 8-order & 9-order \\
\hline 1981 & 1025 & V_high & & & & & & & & \\
\hline 1982 & 512 & V_low & & & & & & & & \\
\hline 1983 & 1005 & V_high & 1016 & & & & & & & \\
\hline 1984 & 852 & L_high & 837 & 837 & & & & & & \\
\hline 1985 & 440 & A_low & 400 & 400 & 400 & & & & & \\
\hline 1986 & 502 & V_low & 484 & 484 & 484 & 484 & & & & \\
\hline 1987 & 775 & Medium & 750 & 750 & 750 & 750 & 750 & & & \\
\hline 1988 & 465 & V_low & 484 & 484 & 484 & 484 & 484 & 484 & & \\
\hline 1989 & 795 & L_high & 837 & 837 & 837 & 837 & 837 & 837 & 837 & \\
\hline 1990 & 970 & high & 929 & 929 & 929 & 929 & 929 & 929 & 929 & 929 \\
\hline 1991 & 742 & medium & 750 & 750 & 750 & 750 & 750 & 750 & 750 & 750 \\
\hline 1992 & 635 & L_low & 663 & 663 & 663 & 663 & 663 & 663 & 663 & 663 \\
\hline 1993 & 994 & V_high & 1016 & 1016 & 1016 & 1016 & 1016 & 1016 & 1016 & 1016 \\
\hline 1994 & 759 & medium & 750 & 750 & 750 & 750 & 750 & 750 & 750 & 750 \\
\hline 1995 & 883 & high & 929 & 929 & 929 & 929 & 929 & 929 & 929 & 929 \\
\hline 1996 & 599 & low & 572 & 572 & 572 & 572 & 572 & 572 & 572 & 572 \\
\hline 1997 & 499 & V_low & 484 & 484 & 484 & 484 & 484 & 484 & 484 & 484 \\
\hline 1998 & 590 & low & 572 & 572 & 572 & 572 & 572 & 572 & 572 & 572 \\
\hline 1999 & 911 & high & 929 & 929 & 929 & 929 & 929 & 929 & 929 & 929 \\
\hline 2000 & 862 & L_high & 837 & 837 & 837 & 837 & 837 & 837 & 837 & 837 \\
\hline 2001 & 801 & L_high & 837 & 837 & 837 & 837 & 837 & 837 & 837 & 837 \\
\hline 2002 & 1067 & A_high & 1100 & 1100 & 1100 & 1100 & 1100 & 1100 & 1100 & 1100 \\
\hline 2003 & 917 & high & 929 & 929 & 929 & 929 & 929 & 929 & 929 & 929 \\
\hline \multicolumn{2}{|l|}{ MSE } & & 713.6 & 743.3 & 770.5 & 724.4 & 748 & 755.7 & 782 & 711.9 \\
\hline \multicolumn{2}{|l|}{ AFE } & & 3.3707 & 3.4846 & 3.5753 & 3.2689 & 3.2502 & 3.2518 & 3.1961 & 3.0471 \\
\hline
\end{tabular}


TABLE X. COMPARISON OF MSE AND AFE VALUES

\begin{tabular}{|c|c|c|c|c|c|c|c|c|c|}
\hline Evaluation method & Forecasting models & 2-order & 3-order & 4-order & 5-order & 6-order & 7-order & 8-order & 9-order \\
\hline \multirow{2}{*}{ MSE } & Singh [17] & 1089.7 & 908.9 & 1191.5 & 1311.6 & 1597.0 & 1207.2 & 1259.9 & 1425.8 \\
\hline & Proposed & 713.6 & 743.3 & 770.5 & 724.4 & 748 & 755.7 & 782 & 711.9 \\
\hline \multirow{2}{*}{ AFE } & Singh [17] & 4.1359 & 3.8137 & 4.3566 & 4.4677 & 4.8753 & 3.9747 & 4.1337 & 4.5543 \\
\hline & Proposed & 3.3707 & 3.4846 & 3.5753 & 3.2689 & 3.2502 & 3.2518 & 3.1961 & 3.0471 \\
\hline
\end{tabular}

\section{CONCLUSIONS}

The aims of the study in this paper is to establish a formalized methodology for handling words directly to solve the time series forecasting problems in such a way that it can simulate the way human users making a forecasting process in terms of their words. In fact, when observing the numerical time series data, human users can quickly transform its variations into the word variations which form a linguistic time series. They can do reasoning in their mind in some ways based on the relationships of the words in linguistic time series to estimate the forecasting values.

This paper focuses on examining the high-order linguistic time series to establish an efficient linguistic forecasting method in such a way that a formal formalism is developed to ensure that the inherent semantics of words, both qualitative and quantitative, are defined in the full linguistic variable context and the declared word-set of the linguistic variable can grow to increase the forecasted results. The growing of the declared word-set means that the human domain knowledge is grown. It is true in practice because human knowledge grows day by day. So, when human experts want to increase the forecasted results, he can make the declared word-set grow whilst keeps all semantic types of the existing words unchanged.

The proposed forecasting method is applied to the forecasting problems of the enrollments of University of Alabama and Lahi crop production of India. The experimental results have shown that the proposed forecasting method is better than the ones proposed by Song and Chissom, Chen, Hwang and Singh and the growth of the declared word-set of the linguistic variable is significant in increasing the accuracy of forecasted results of the high-order linguistic forecasting method. Therefore, that proves the effectiveness of our proposed approach.

\section{REFERENCES}

[1] L. A. Zadeh, "Fuzzy Sets,” Inf. Control, vol. 8, pp. 338-353, 1965, doi: 10.1016/S0019-9958(65)90241-X.

[2] Q. Song and B. S. Chissom, "Fuzzy time series and its models," Fuzzy Sets Syst., vol. 54, pp. 269-277, 1993.

[3] Q. Song and B. S. Chissom, "Forecasting enrollments with fuzzy time series - part 1,” Fuzzy Sets Syst., vol. 54, pp. 1-9, 1993.

[4] Q. Song and B. S. Chissom, "Forecasting enrollments with fuzzy time series - part 2,” Fuzzy Sets Syst., vol. 62, pp. 1-8, 1994.

[5] S. M. Chen, "Forecasting enrollments based on high-order fuzzy time series," Cybern. Syst., vol. 33, no. 1, pp. 1-16, 2002, doi: 10.1080/019697202753306479.

[6] S.-M. Chen and N.-Y. Chung, "Forecasting Enrollments Using HighOrder Fuzzy Time Series and Genetic Algorithms,” Int. J. Intell. Syst., vol. 21, pp. 485-501, 2006.

[7] L.-W. Lee, S.-M. Chen, Y.-H. Leu, and L.-H. Wang, "Handling Forecasting Problems Based on Two-Factors High-Order Fuzzy Time Series,” IEEE Trans. Fuzzy Syst., vol. 14, p. 10, 2006.
[8] K. K. Gupta and S. Kumar, "A novel high-order fuzzy time series forecasting method based on probabilistic fuzzy sets," Granul. Comput., no. 2016, 2019, doi: 10.1007/s41066-019-00168-4.

[9] P. Jiang, Q. Dong, P. Li, and L. Lian, “A novel high-order weighted fuzzy time series model and its application in nonlinear time series prediction,” Appl. Soft Comput., vol. 55, pp. 44-62, 2017, doi: 10.1016/j.asoc.2017.01.043.

[10] M. Bose and K. Mali, "A novel data partitioning and rule selection technique for modeling high-order fuzzy time series," Appl. Soft Comput., vol. 63, pp. 87-96, 2018, doi: 10.1016/j.asoc.2017.11.011.

[11] E. Bas, C. Grosan, E. Egrioglu, and U. Yolcu, "High order fuzzy time series method based on pi-sigma neural network," Eng. Appl. Artif. Intell., vol. 72, pp. 350-356, Jun. 2018, doi: 10.1016/j.engappai.2018.04.017.

[12] P. Singh and Y.-P. Huang, "A High-Order Neutrosophic-Neuro-Gradient Descent Algorithm-Based Expert System for Time Series Forecasting," Int. J. Fuzzy Syst., 2019, doi: 10.1007/s40815-019-00690-2.

[13] S. Panigrahi and H. S. Behera, "A study on leading machine learning techniques for high order fuzzy time series forecasting," Eng. Appl. Artif. Intell., vol. 87, no. November 2019, p. 103245, 2020, doi: 10.1016/j.engappai.2019.103245.

[14] R. M. Pattanayak, S. Panigrahi, and H. S. Behera, "High-Order Fuzzy Time Series Forecasting by Using Membership Values Along with Data and Support Vector Machine,” Arab. J. Sci. Eng., no. 1, 2020, doi: 10.1007/s13369-020-04721-1.

[15] R. Mohan, H. S. Behera, and S. Panigrahi, "A novel probabilistic intuitionistic fuzzy set based model for high order fuzzy time series forecasting Engineering Applications of Artificial Intelligence A novel probabilistic intuitionistic fuzzy set based model for high order fuzzy time series forecas,” Eng. Appl. Artif. Intell., vol. 99, no. March, p. 104136, 2021, doi: 10.1016/j.engappai.2020.104136.

[16] E. Egrioglu, C. H. Aladag, U. Yolcu, V. R. Uslu, and M. A. Basaran, “A new approach based on artificial neural networks for high order multivariate fuzzy time series," Expert Syst. Appl., vol. 36, no. 7, pp. 10589-10594, 2009, doi: 10.1016/j.eswa.2009.02.057.

[17] S. R. Singh, "A computational method of forecasting based on high-order fuzzy time series,” Expert Syst. Appl., vol. 36, no. 7, pp. 10551-10559, 2009, doi: 10.1016/j.eswa.2009.02.061.

[18] S. M. Chen and C. D. Chen, "Handling forecasting problems based on high-order fuzzy logical relationships,” Expert Syst. Appl., vol. 38, no. 4, pp. 3857-3864, 2011, doi: 10.1016/j.eswa.2010.09.046.

[19] C. H. Aladag, U. Yolcu, and E. Egrioglu, “A high order fuzzy time series forecasting model based on adaptive expectation and artificial neural networks,” Math. Comput. Simul., vol. 81, no. 4, pp. 875-882, 2010, doi: 10.1016/j.matcom.2010.09.011.

[20] S. S. Gangwar and S. Kumar, "Partitions based computational method for high-order fuzzy time series forecasting,” Expert Syst. Appl., vol. 39, no. 15, pp. 12158-12164, 2012, doi: 10.1016/j.eswa.2012.04.039.

[21] M. Y. Chen, "A high-order fuzzy time series forecasting model for internet stock trading,” Futur. Gener. Comput. Syst., vol. 37, pp. 461467, 2014, doi: 10.1016/j.future.2013.09.025.

[22] S. M. Chen and S. W. Chen, "Fuzzy forecasting based on two-factors second-order fuzzy-trend logical relationship groups and the probabilities of trends of fuzzy logical relationships,” IEEE Trans. Cybern., vol. 45, no. 3, pp. 405-417, 2015, doi: 10.1109/TCYB.2014.2326888.

[23] E. Egrioglu, C. H. Aladag, U. Yolcu, and A. Z. Dalar, "A Hybrid High Order Fuzzy Time Series Forecasting Approach Based on PSO and ANNs Methods,” Am. J. Intell. Syst., vol. 6, no. 1, p. 8, 2016.

[24] N. C. Ho and W. Wechler, "Hedge Algebras: An algebraic approach to structure of sets of linguistic truth values," Fuzzy Sets Syst., vol. 35, pp. 281-293, 1990. 
[25] N. C. Ho, V. N. Lan, and L. X. Viet, "Optimal hedge-algebras-based controller: Design and application,” Fuzzy Sets Syst., vol. 159, p. 32, 2008.

[26] C. H. Nguyen, D. A. Nguyen, N. L. Vu, and D. A. Nguyen, "Fuzzy Controller using Hedge Algebra based semantics of vague linguistic terms," in Fuzzy Control Systems, D. Vukadinovic, Ed. Nova Science Publishers, Inc., 2011.

[27] D. Vukadinovic, M. Basic, C. H. Nguyen, N. L. Vu, and T. D. Nguyen, "Hedge-algebra-based voltage controller for a self-excited induction generator,” Control Eng. Pract., vol. 30, pp. 78-90, 2014.

[28] H.-L. Bui, C.-H. Nguyen, V.-B. Bui, K.-N. Le, and H.-Q. Tran, "Vibration control of uncertain structures with actuator saturation using hedge-algebras-based fuzzy controller,” J. Vib. Control, p. 19, 2015.

[29] D. T. Tran, V. B. Bui, T. A. Le, and H. Le Bui, "Vibration control of a structure using sliding-mode hedge-algebras-based controller," Soft Comput., vol. 23, no. 6, pp. 2047-2059, 2017.

[30] H. Le Bui, T. A. Le, and V. B. Bui, "Explicit formula of hedge-algebrasbased fuzzy controller and applications in structural vibration control," Appl. Soft Comput., vol. 60, pp. 150-166, 2017.

[31] H.-L. Bui, N.-L. Vu, C.-H. Nguyen, and C.-H. Nguyen, "General design method of hedge-algebras-based fuzzy controllers and an application for structural active control,” Appl. Intell., vol. 43, p. 25, 2015.

[32] C. H. Nguyen, T. S. Tran, and D. P. Pham, "Modeling of a semantics core of linguistic terms based on an extension of hedge algebra semantics and its application,” Knowledge-Based Syst., vol. 67, p. 19, 2014.
[33] C. H. Nguyen, V. T. Hoang, and V. L. Nguyen, "A discussion on interpretability of linguistic rule based systems and its application to solve regression problems,” Knowledge-Based Syst., vol. 88, pp. 107-133, 2015.

[34] H. H. Ngo, C. H. Nguyen, and V. Q. Nguyen, "Multichannel image contrast enhancement based on linguistic rule-based intensificators," Appl. Soft Comput., vol. 76, pp. 744-763, 2019.

[35] N. D. Hieu, N. C. Ho, and V. N. Lan, "Enrollment Forecasting Based on Linguistic Time Series,” J. Comput. Sci. Cybern., vol. 36, no. 2, pp. 119137, 2020, doi: 10.15625/1813-9663/36/2/14396.

[36] N. D. Hieu, N. C. Ho, and V. N. Lan, "An efficient fuzzy time series forecasting model based on quantifying semantics of words," in IEEE RIVF International Conference, 2020, pp. 1-6.

[37] N. C. Ho and W. Wechler, "Extended hedge algebras and their application to fuzzy logic,” Fuzzy Sets Syst., vol. 52, pp. 259-281, 1992.

[38] N. C. Ho and N. Van Long, "Fuzziness measure on complete hedge algebras and quantifying semantics of terms in linear hedge algebras," Fuzzy Sets Syst., vol. 158, pp. 452-471, 2007.

[39] J. R. Hwang, S. M. Chen, C. H. Lee, Handling forecasting problems using fuzzy time series. Fuzzy Sets and Systems, vol. 100, pp. 217-228, 1998. 